\title{
氮杂穴醚配体及其金属配合物对小分子的 识别及活化作用
}

\author{
陈嘉媚 鲁统部* \\ (中山大学化学与化学工程学院, 广州 510275.*联系人, E-mail: lutongbu@mail.sysu.edu.cn)
}

\begin{abstract}
摘要 分子识别定义为主体分子对客体分子的选择性结合过程. 有选择性地识别小分子一直是超分子 化学中具有挑战性的研究课题. 氮杂穴醚配体及其金属配合物作为典型的主体分子得到了广泛的研究. 本文总结了氮杂穴醚配体及其金属配合物对各种小分子的识别作用，介绍了新近报道的氮杂穴醚双核 金属配合物在基于分子识别的基础上对小分子的活化作用，展望了该领域的发展趋势.
\end{abstract}

关键词 氮杂穴醚配体 双核金属配合物 分子识别与活化

自从 20 世纪 70 年代Lehn首次合成出氮杂穴醚配 体以来 $[1]$, 科学家们在这个领域上已经研究了 30 余年. 氮杂穴醚配体及其金属配合物由于其独特的 配位特性, 在小分子识别方面具有较好的研究前 景 ${ }^{[2 \sim 6]}$. 分子识别定义为主体分子对客体分子的选择 性结合过程. 有选择性地识别小分子, 一直是超分子 化学中具有挑战性的课题 ${ }^{[7-11]}$. 此外, 分子识别是生 物酶催化底物反应的前提和基础, 生物酶首先要对 底物分子发生多位点的识别作用使其活化，然后在 温和的条件下即可催化底物发生反应. 氮杂穴醚双 核金属配合物具有双金属中心结构, 这与生物体内 许多金属酶，如铜锌超氧化物歧化酶、单加氧酶、尿 素酶、氨肽酶、磷酸酯酶等的双金属活性中心非常相 似 $[12,13]$. 氮杂穴醚双核金属配合物能与反应底物配 位，这种配位作用及穴醚配体空腔的空间保护作用 同样可以使反应底物活化, 具有类似生物酶的催化 活性, 从而使其在小分子催化活化方面也具有不俗 的表现.

\section{1 氮杂穴醚配体及其金属配合物}

氮杂穴醚配体是由 3 个间隔基团 $\mathrm{R}$ 连接两个桥头 $\mathrm{N}$ 原子形成的双环配体. 氮杂穴醚配体种类多样, 主 要是由二醛基或二酮基化合物与三(2-乙基胺)氨(tren) 缩合形成希夫碱配合物后还原得到的 ${ }^{[14]}$ (图 1). 通过 改变连接两端tren的间隔基团 $\mathrm{R}$, 我们可以控制生成 不同尺寸和刚性的孔穴, 从而可以得到结构相异、功 能不同的穴醚配体. 在早期的研究中, 间隔基团 $\mathrm{R}$ 多

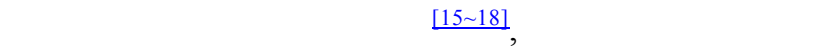

较好, 容易变形; 以烯烃或炔烃作为间隔基团 $\mathrm{R}$ 的配 体也有报道 ${ }^{[19-21]}$, 这类配体刚性较强, 链烃两头的 $\mathrm{N}$ 原子由于连接不饱和烃而碱性较弱. 近年来, 研究多 的是以五元或六元环的刚性基团, 如苯环、呋喃环、

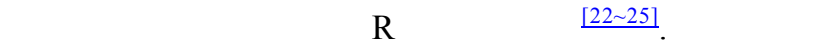
基团体积大, 刚性强, 增强了环内空腔的刚性, 从而 提高了其识别小分子的选择性, 这类配体是本文的 主要讨论对象.

氮杂穴醚配体两端各含有 4 个 $\mathrm{N}$ 原子的配位空腔, 容易与两个金属离子配位形成双核金属配合物 ${ }^{[2225]}$. 金属离子分别与 4 个 $\mathrm{N}$ 原子配位后, 在轴向各空出了 一个配位点, 并且刚性的环状间隔基团限定了两个 金属离子之间的距离, 从而很容易选择性地接受其 他与之相匹配的小分子基团, 如卤素, $\mathrm{CN}^{-}, \mathrm{CO}_{3}{ }^{2-}$, $\mathrm{N}_{3}^{-}, \mathrm{SCN}^{-}, \mathrm{NO}_{3}{ }^{-}$等的配位, 形成桥联的双核金属配合 物 ${ }^{[22 ~ 24]}$, 实现对不同小分子的选择性识别.

除了通过改变间隔基团 $\mathrm{R}$ 使氮杂穴醚配体的结 构发生变化之外, 人们也经常通过对氮杂穴醚配体 中某些基团进行修饰(如引入支链)得到结构、功能不 同的大环配体. 例如在间隔基团苯环上引入羟基、巯 基, 在仲胺的 $N$ 原子上引入甲基或乙基等等 ${ }^{[26 ~ 36]}$. 这 样, 由于配位环境的变化, 使得相应的金属配合物的 性质与功能也会发生改变.

\section{2 质子化的氮杂穴醚配体对阴离子的识别 作用}

氮杂穴醚配体本身显碱性, 6 个仲胺质子化后形 成一个电正性的配位空腔, 其中每个质子化的仲胺 


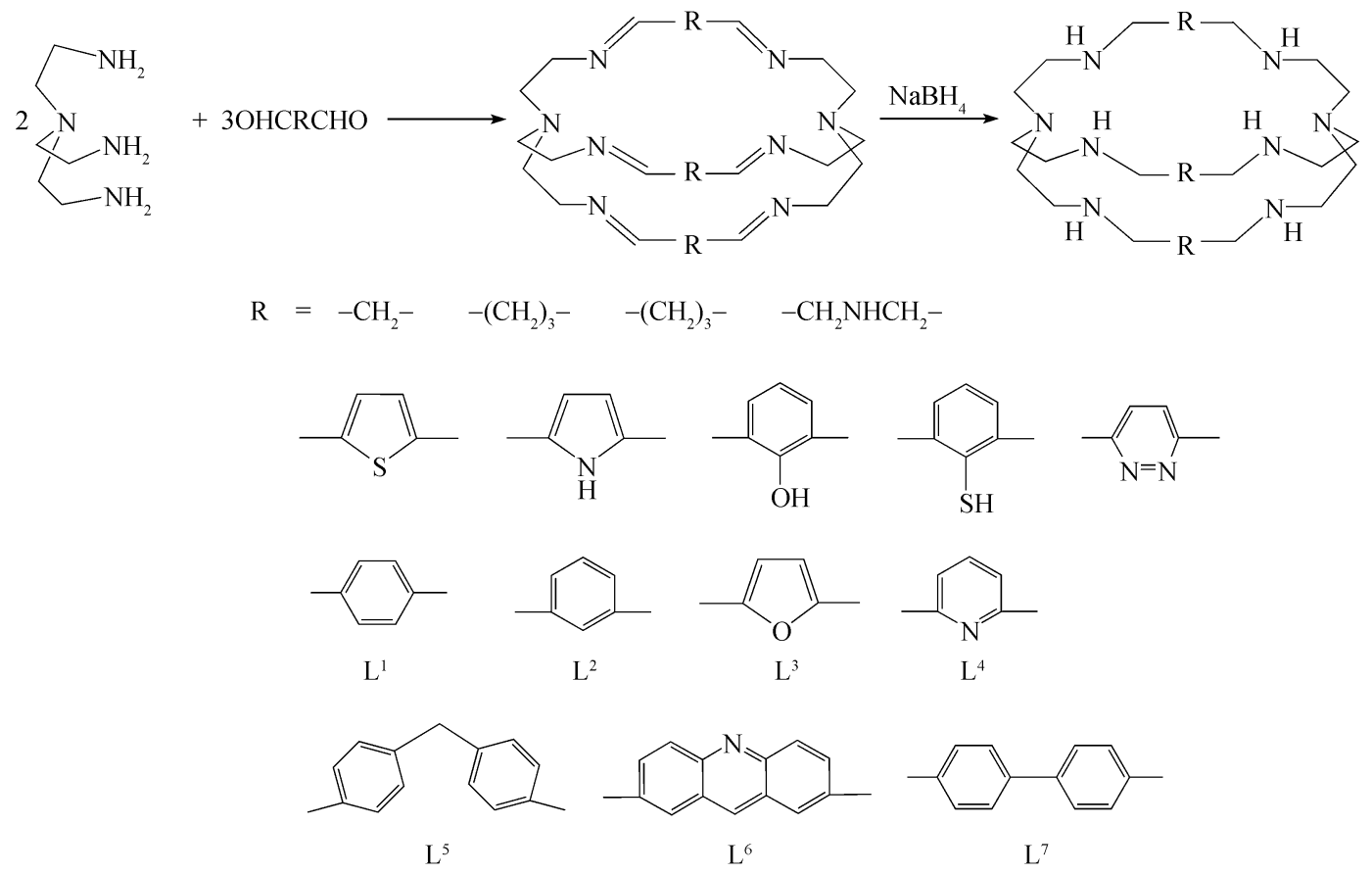

图 1 氮杂穴醚配体及其合成方法

基团可以作为氢键的给体与客体分子同时形成两个 氢键，在适当的条件下，客体分子与芳香的间隔基团 之间也可以发生 $\pi \cdots \pi$ 堆积作用. 所以质子化的氮杂 穴醚配体可以利用静电作用、氢键作用和 $\pi \cdots \pi$ 堆积 作用结合各种阴离子 ${ }^{[3]}$. 由于间隔基团 R不同，不同 的氮杂穴醚配体对于各种阴离子的结合具有一定的 选择性.

以对位苯环作为间隔基团 $\mathrm{R}$ 的穴醚配体 $\left[\mathrm{H}_{6} \mathrm{~L}^{1}\right]^{6+}$, 由于 3 个苯环两两之间贴得较近, 使得大阴离子难以 进入, 只能包结卤素 ${ }^{[37,38]}$ 或 $\mathrm{NO}_{3}{ }^{-[2]}$ 这类较小的阴离 子. 晶体结构研究结果表明 ${ }^{[37]},\left[\mathrm{H}_{6} \mathrm{~L}^{1}\right]^{6+}$ 与 $\mathrm{F}^{-}$作用时, 两个 $\mathrm{F}^{-}$同时占据穴醚配体孔穴的两端, 之间通过氢 键与一个水分子桥联(图 2(a)). 这一桥联结构类似于 穴醚桥联双核金属配合物. $\left[\mathrm{H}_{6} \mathrm{~L}^{1}\right]^{6+}$ 与 $\mathrm{Cl}^{-}, \mathrm{Br}^{-}$存在两 种不同的结合方式: (1) 一个卤素离子和一个水分子 分别占据穴醚配体孔穴两端的配位点, 卤素离子和 水分子之间有弱的氢键作用, 两端的叔氮的距离分 别是 $10.039 \AA\left(\mathrm{Cl}^{-}\right)$(图 2(b))和 $10.377 \AA\left(\mathrm{Br}^{-}\right)^{[37]}$. (2) 个卤素离子占据穴醚配体孔穴的中心, 两端的叔氮 的距离分别是 $6.240 \AA\left(\mathrm{Cl}^{-}\right)$(图 2(c))和 $6.486 \AA\left(\mathrm{Br}^{-}\right)^{[38]}$. 而 $\mathrm{I}^{-}$体积太大, 无法进入穴醚配体 $\left[\mathrm{H}_{6} \mathrm{~L}^{1}\right]^{6+}$ 的孔穴, 只 能在外围以抗衡离子的形式存在. 根据核磁滴定 ${ }^{[37]},\left[\mathrm{H}_{6} \mathrm{~L}^{1}\right]^{6+}$ 与 $\mathrm{F}^{-}, \mathrm{Cl}^{-}, \mathrm{Br}^{-}$在 $\mathrm{pH}=5$ 的水溶液中的结合
常数 $\log K$ 值分别为 $3.15(5), 3.37(3)$ 和 3.34(4), 虽然结 合作用较强, 但没有明显的选择性. 最近一篇报道显 示 ${ }^{[39]}$, 含 4 个质子的 $\left[\mathrm{H}_{4} \mathrm{~L}^{1}\right]^{4+}$ 两端各有两个仲胺质子 化(即图 2(d)中的N2, N5, N3, N8), 两个水分子通过 氢键分别与两端结合，同时两个水分子之间通过氢 键与一个乙腈分子桥联, 所以 $\left[\mathrm{H}_{4} \mathrm{~L}^{1}\right]^{4+}$ 结合了水 $\cdots$ 乙 腈…水这样一个三分子簇. 而含 3 个质子的 $\left[\mathrm{H}_{3} \mathrm{~L}^{1}\right]^{3+}$ 一端的叔胺和另一端的两个仲胺质子化(即图 2(e)中 的N4, N5, N7), 只有含两个质子化仲胺的一端结合 一个水分子. 由此可见, 可以通过调节 $\mathrm{L}^{1}$ 的质子化程 度改变其结合小分子客体的性质.

相比之下，以间位苯环作为间隔基团 $\mathrm{R}$ 的穴醚配 体 $\left[\mathrm{H}_{6} \mathrm{~L}^{2}\right]^{6+}$ 可称为较 “慷慨”的配体，它不仅能结合 $\mathrm{F}^{-[40]}, \mathrm{NO}_{3}{ }^{-[41,42]}$, 还能容纳四面体的 $\mathrm{ClO}_{4}{ }^{-[42]}, \mathrm{ReO}_{4}{ }^{-[3]}$ 以及二价阴离子 $\mathrm{SO}_{4}{ }^{2-[43]}, \mathrm{SeO}_{4}{ }^{2-}, \mathrm{CrO}_{4}{ }^{2-}, \mathrm{S}_{2} \mathrm{O}_{3}{ }^{2-[44]}$ 和 $\mathrm{C}_{2} \mathrm{O}_{4}{ }^{2-[45]}$ 等等. 根据晶体结构, $\left[\mathrm{H}_{6} \mathrm{~L}^{2}\right]^{6+}$ 与 $\mathrm{F}^{-}$的结合方 式类似于 $\left[\mathrm{H}_{6} \mathrm{~L}^{1}\right]^{6+}$ 与 $\mathrm{Cl}^{-}, \mathrm{Br}^{-}$的第一种结合方式, 即一 个 $\mathrm{F}^{-}$和一个水分子分别占据穴醚配体孔穴两端的配 位点, $\mathrm{F}^{-}$和水分子之间存在氢键作用 $[40]$ (图 $3(\mathrm{a})) .\left[\mathrm{H}_{6} \mathrm{~L}^{2}\right]^{6+}$ 与 $\mathrm{NO}_{3}{ }^{-}$作用时，可以同时包结两个相 互平行的 $\mathrm{NO}_{3}^{-[41,42]}$ (图 3(b)). 电位滴定和核磁滴定 ${ }^{[42]}$ 结果表明, 当 $\mathrm{NO}_{3}{ }^{-}$浓度较低时, 主要形成 $1: 1$ 的结合 物, 结合常数 $\log K$ 在 $3 \sim 4$ 之间; 只有在 $\mathrm{NO}_{3}^{-}$ 


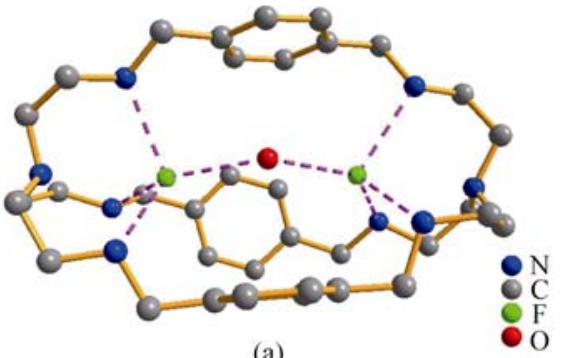

(a)

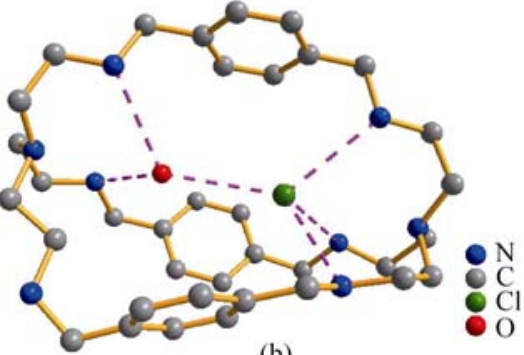

(b)

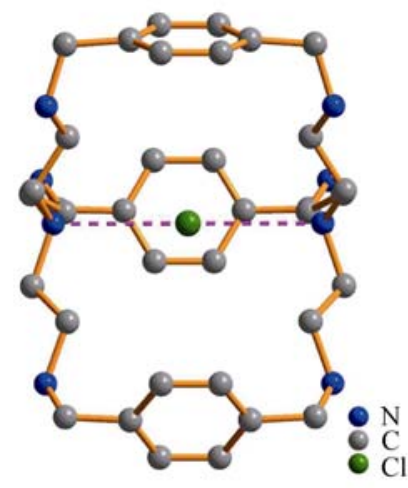

(c)

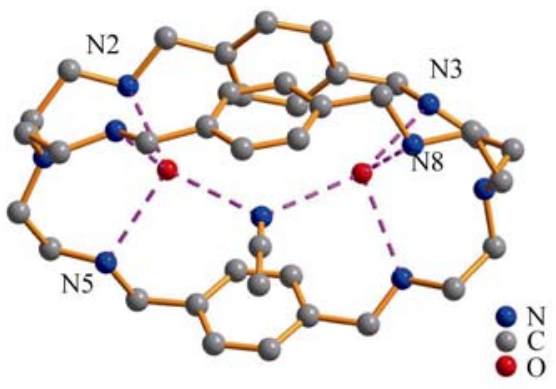

(d)

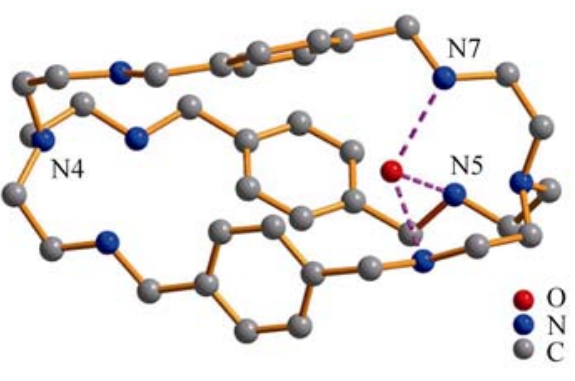

(e)

图 2 大环阳离子结合阴离子及溶剂分子的晶体结构图

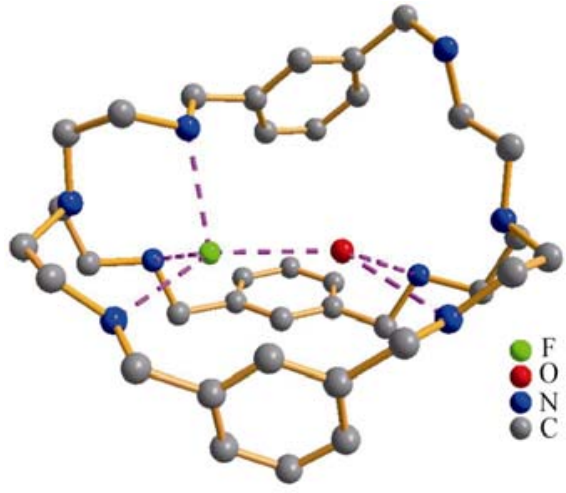

(a)

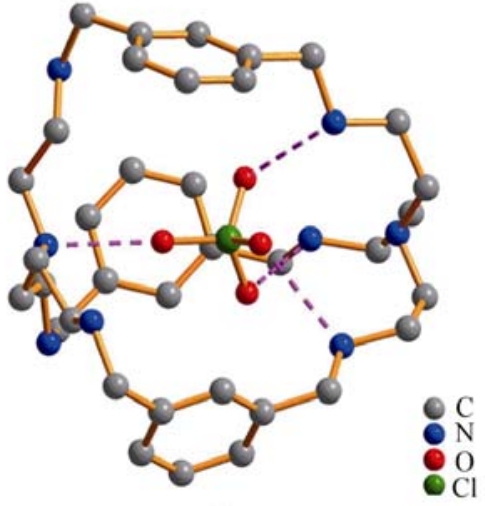

(c)

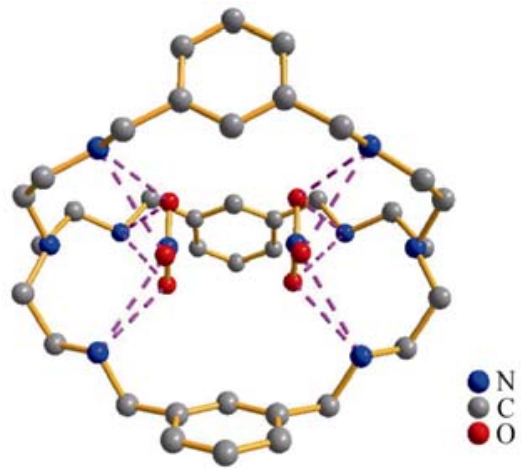

(b)

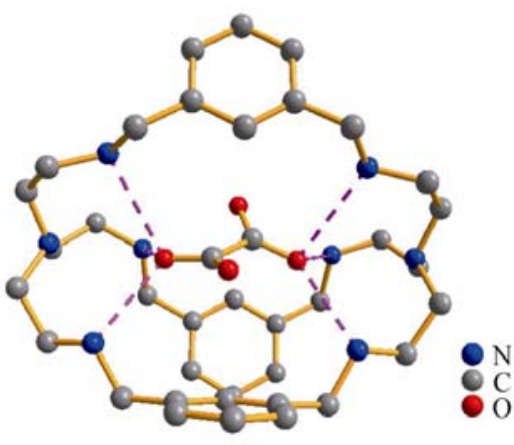

(d)

图 $3\left[\mathrm{H}_{6} \mathrm{~L}^{2}\right]^{6+}$ 结合阴离子的晶体结构图 
浓度大大过量于 $\left[\mathrm{H}_{6} \mathrm{~L}^{2}\right]^{6+}$ 浓度时, 才有 $1: 2$ 的结合物 生成, 其二级结合常数 $K_{2}=1.87 \pm 0.73$. 由于阴离子 结合物的稳定性主要是由静电作用决定的, 因此比 较质子化穴醚配体对具有相似结构不同价态的阴离 子的结合能力颇具意义. 初步的研究结果表明: $\left[\mathrm{H}_{6} \mathrm{~L}^{2}\right]^{6+}$ 与 $\mathrm{ClO}_{4}{ }^{-}$在溶液中的结合常数太小, 无法测出精 确的数值; 而 $\left[\mathrm{H}_{6} \mathrm{~L}^{2}\right]^{6+}$ 与 $\mathrm{SeO}_{4}^{2-}, \mathrm{S}_{2} \mathrm{O}_{3}^{2-}$ 的结合常数大约 在 $\log K=4.0(1)$ 左右, 与 $\mathrm{SO}_{4}^{2-}$ 的结合常数 $\log K=$ 4.43(1). 由于静电作用增强, $\left[\mathrm{H}_{6} \mathrm{~L}^{2}\right]^{6+}$ 与阴离子的结合 能力显著增强. 由此可见, 相比于一价阴离子, 质子 化的穴醚配体对于二价阴离子具有更好的动力学稳 定性和选择性. 此外, $\left[\mathrm{H}_{6} \mathrm{~L}^{2}\right]^{6+}$ 与 $\mathrm{C}_{2} \mathrm{O}_{4}^{2-}$ 作用时, $\mathrm{C}=\mathrm{O}$ 双键与苯环之间的距离小于 $3.6 \AA$, 这表明羧基与苯 环之间存在 $\pi \cdots \pi$ 堆积作用, 这种作用导致 $\mathrm{C}_{2} \mathrm{O}_{4}^{2-}$ 的平 面构型发生扭曲 ${ }^{[45]}$ (图 3(d)). $\left[\mathrm{H}_{6} \mathrm{~L}^{2}\right]^{6+}$ 与 $\mathrm{C}_{2} \mathrm{O}_{4}^{2-}$ 的结合 常数 $\log K=10.50(8)$, 远远高于其他二价阴离子, 这 说明在结合阴离子时 $\pi \cdots \pi$ 堆积作用对于稳定性的贡 献也很重要.

质子化的穴醚配体 $\left[\mathrm{H}_{6} \mathrm{~L}^{3}\right]^{6+}$ 也可以包结含氧阴离 子, 如 $\mathrm{ClO}_{4}^{-[46]}, \mathrm{C}_{2} \mathrm{O}_{4}^{2-[45]}$ (图 4)等等. 但是相对于 $\left[\mathrm{H}_{6} \mathrm{~L}^{2}\right]^{6+},\left[\mathrm{H}_{6} \mathrm{~L}^{3}\right]^{6+}$ 与阴离子的结合常数较小, 这可能 是由于以呋喃环作为间隔基团的穴醚配体碱性较弱 $\left(\mathrm{L}^{3}\right.$ 的 $\Sigma K_{\mathrm{i}}=43.46, \mathrm{~L}^{2}$ 的 $\left.\Sigma K_{\mathrm{i}}=46.76\right)$ 的原因造成的.

以吡啶作为间隔基团 $\mathrm{R}$ 的穴醚配体 $\mathrm{L}^{4}$ 的碱性与 $\mathrm{L}^{2}$ 相当, $\Sigma K_{\mathrm{i}}=45.36^{[3]}$. 与 $\mathrm{L}^{2}$ 和 $\mathrm{L}^{3}$ 相比, $\mathrm{L}^{4}$ 倾向于与阴 离子以包夹的形式结合: 即一个或多个阴离子结合 在 3 个吡啶环两两形成的凹槽之间. 这可能是因为以 这种包夹的形式结合时可以利用吡啶环上的氮原子

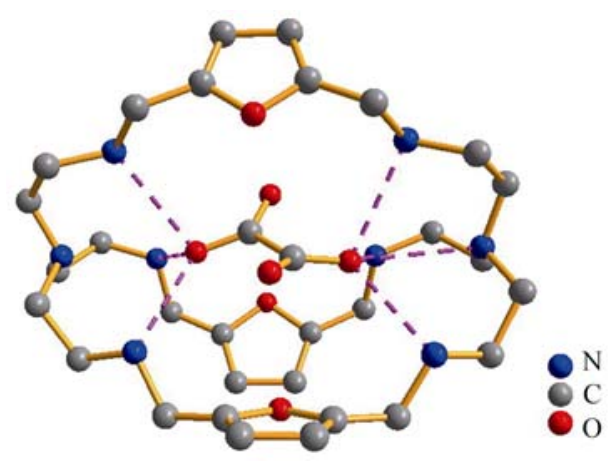

图 $4 \quad\left[\mathrm{H}_{6} \mathrm{~L}^{3}\right]^{6+}$ 结合阴离子的晶体结构图

生成氢键, 同时有利于阴离子与吡啶环之间发生 $\pi \cdots \pi$ 堆积作用. 例如当 $\left[\mathrm{H}_{8} \mathrm{~L}^{4}\right]^{8+}$ 与 $\mathrm{ClO}_{4}{ }^{-}$结合时 ${ }^{[47]}$, 两个吡 啶环上的 $\mathrm{N}$ 原子也质子化参与形成氢键 (图 5(a)). $\left[\mathrm{H}_{6} \mathrm{~L}^{4}\right]^{6+}$ 与 $\mathrm{C}_{2} \mathrm{O}_{4}{ }^{2-}$ 结合时 ${ }^{[3]}, \mathrm{C}_{2} \mathrm{O}_{4}{ }^{2-}$ 的一端结合 在穴醚配体的凹槽内, 另一端指向外面, 质子化参与 形成氢键, $\mathrm{C}_{2} \mathrm{O}_{4}{ }^{2-}$ 相互之间形成的这种氢键使得穴醚 化合物以二聚体的形式成对存在(图 5(b)). $\mathrm{C}_{2} \mathrm{O}_{4}{ }^{2-}$ 与 $\left[\mathrm{H}_{6} \mathrm{~L}^{4}\right]^{6+}$ 结合时的强相互作用使其结合常数非常高, 甚至可以成功地和阳离子如 $\mathrm{Fe}^{2+}$ 或 $\mathrm{Ca}^{2+}$ 竞争配位 ${ }^{[3]}$.

氮杂穴醚配体 $\mathrm{L}^{5}, \mathrm{~L}^{6}$ (图 1)的孔穴呈较长的椭球 状, Lehn小组 ${ }^{[48,49]}$ 研究了 $\left[\mathrm{H}_{6} \mathrm{~L}^{5}\right]^{6+},\left[\mathrm{H}_{6} \mathrm{~L}^{6}\right]^{6+}$ 与链状阴 离子 ${ }^{-} \mathrm{OOC}-\left(\mathrm{CH}_{2}\right)_{n}-\mathrm{COO}^{-}(n=1 \sim 7)$ 的结合作用. 研究 结果表明, $\left[\mathrm{H}_{6} \mathrm{~L}^{5}\right]^{6+}$ 由于两个苯环之间的亚甲基使配 体的柔韧性较大, 与二羧酸根阴离子结合没有明显 的选择性 ${ }^{[48]}$; 相比之下刚性较强的 $\left[\mathrm{H}_{6} \mathrm{~L}^{6}\right]^{6+}$ 对二羧酸 根阴离子具有较强的识别作用 ${ }^{[49]}$ : 当 $n=1,2$ 时, 阴 离子长度与配体的孔穴不匹配, 基本上没有结合作

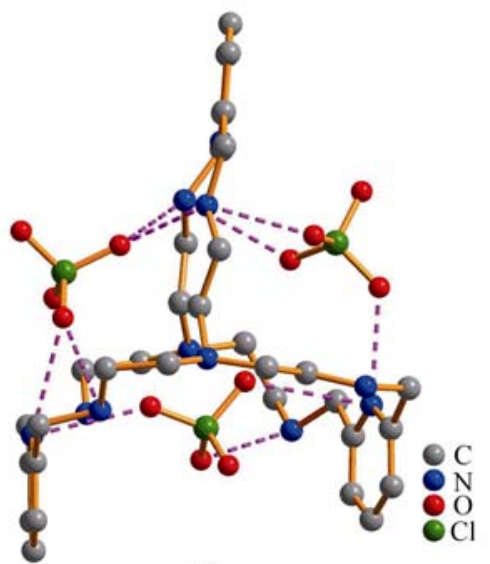

(a)

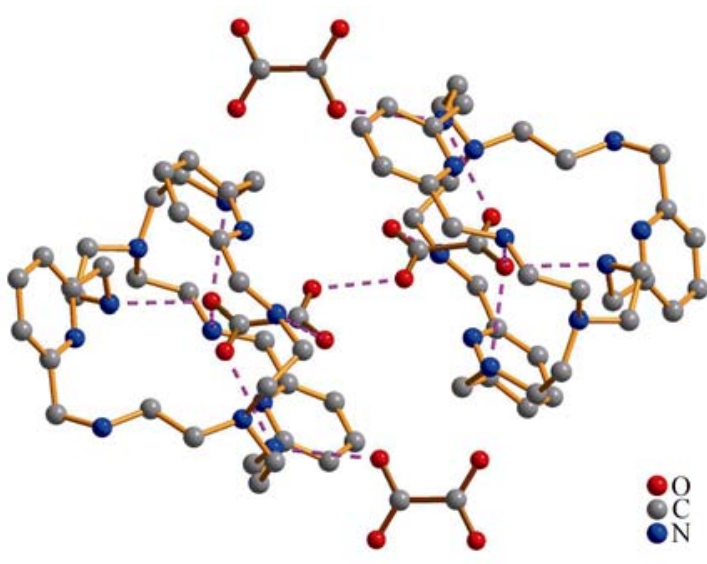

(b)

图 5.s $\left[\mathrm{H}_{6} \mathrm{~L}^{4}\right]^{6+}$ 结合阴离子的晶体结构图 52-4-369 
用; 当 $n \geq 3$ 时, 其结合常数 $\log K$ 值分别为 $2.7(n=$ $3), 3.6(n=4)$ 和 $3.2(n=5)$, 对羒酸链的长度具有明显 的识别作用. $\left[\mathrm{H}_{6} \mathrm{~L}^{6}\right]^{6+}$ 与芳香族羧酸根阴离子同样具 有较强的识别作用: 与苯甲酸根阴离子基本上没有 结合; 与邻、间、对苯二甲酸根阴离子的结合常数 $\log K$ 值分别为 $3.5,4.1$ 和 4.2 .

虽然质子化的穴醚配体对各种阴离子具有一定 的结合作用, 但其结合作用主要为静电作用以及作 用力较弱的氢键和 $\pi \cdots \pi$ 堆积作用, 所以在通常情况 下对阴离子的识别作用较弱.

\section{3 氮杂穴醚双核金属配合物对阴离子的 识别作用}

阴离子可以作为配体与金属(特别是 $\mathrm{d}$ 区过渡金 属)配合物配位, 所以金属配合物也可以作为识别阴 离子的主体. 这种配位作用比静电作用和氢键作用 更强, 且具有方向性, 因此氮杂穴醚双核金属配合物 对阴离子有较好的识别作用. 作为识别阴离子的主 体，金属配合物中的金属离子提供正电荷，同时金属 离子的配位必须未达到饱和状态，空出一个或多个 配位点进一步接受匹配的阴离子与其配位. 在 +2 价 的 $3 \mathrm{~d}$ 过渡金属离子中, $\mathrm{Cu}^{2+}$ 比较常见, 可以与大多数 的配位原子形成稳定的配位键; 而且 $\mathrm{Cu}^{2+}$ 本身具有 动力学活性, 其配位基团可以快速地相互置换, 反应 活性较高. 氮杂穴醚配体两端的 tren可以分别与两个 $\mathrm{Cu}^{2+}$ 形成四配位结构, 然后通过与另一个匹配的阴离 子配位达到稳定的桥联五配位结构, 这就是所谓的 “cascade效应”" 稳定性由金属中心和氮杂穴醚配体的电子性质决定; 其对阴离子的识别能力与氮杂穴醚配体的立体构型 密切相关. 我们可以通过改变连接两端tren的间隔基 团改变氮杂穴醚配体空腔的大小和形状，以达到识 别各种阴离子的目的.

具有不同间隔基团 $\mathrm{R}$ 的各种氮杂穴醚双核金属 配合物可以识别不同类型和长度的阴离子. 对于间隔 基团为对位苯环的 $\mathrm{L}^{1}$, 由于苯环两两之间贴得较近, 使得体积稍大的阴离子难以进入. 只有线型 $\left(\right.$ 如 $\mathrm{CN}^{-[51]}$, $\mathrm{N}_{3}{ }^{-[52]}$ )(图 7(a)和 7(b))或平面型阴离子(如咪唑) ${ }^{[53]}$ (图 $7(\mathrm{c}))$ 可以从配位空腔的窗口进入，与金属离子配位. 咪唑与双核铜配合物除了配位作用之外, 咪唑环和 上下两个苯环平行, 距离分别为 $3.21 \AA$ 和 $3.24 \AA$, 存 在强的 $\pi \cdots \pi$ 堆积作用; 咪唑的一个 $\mathrm{C}-\mathrm{H}$ 键与第三个 苯环垂直, 距离为 $3.38 \AA$, 存在 $\mathrm{C}-\mathrm{H} \cdots \pi$ 作用.
穴醚双核铜配合物 $\left[\mathrm{Cu}_{2} \mathrm{~L}^{2}\right]^{4+[54]}$ 能结合各种不同 形状的阴离子: 包括线形的 $\left(\mathrm{N}_{3}^{-}, \mathrm{NCO}^{-}, \mathrm{NCS}^{-}\right)$, 三角 形的 $\left(\mathrm{NO}_{3}^{-}\right), \mathrm{Y}$ 形的 $\left(\mathrm{HCO}_{3}^{-}, \mathrm{CH}_{3} \mathrm{COO}^{-}\right)$以及四面体的 $\left(\mathrm{SO}_{4}^{2-}\right)$. 键合常数从最大的 $\mathrm{N}_{3}^{-}$到最小的 $\mathrm{SO}_{4}^{2-}$ 相差两 个数量级, 具有较好的识别作用, 导致这种选择性识 别的关键因素是阴离子的两个配位原子之间的距离 (bite length). 如果通过 $\log K$ 的对配位原子的距离做 一条曲线(图 8), 我们可以看到一个尖锐的峰, 显示 对 $\mathrm{N}_{3}^{-}, \mathrm{NCO}^{-}$和 $\mathrm{HCO}_{3}^{-}$具有较强的识别作用, 因为它们 与 $\left[\mathrm{Cu}_{2} \mathrm{~L}^{2}\right]^{4+}$ 中两个金属离子轴向配位点之间的距离 最匹配, 能够生成稳定的桥联配合物. 与其他阴离子 结合时穴醚配体的空腔会发生不同程度的扭曲变形, 故生成的桥联配合物不稳定, 结合常数较低.

穴醚双核铜配合物 $\left[\mathrm{Cu}_{2} \mathrm{~L}^{3}\right]^{4+}$ 的配位空腔相对较 短, 可以包结卤素离子形成稳定的桥联配合物 ${ }^{[55]}$ (图 $9(\mathrm{a}))$. 根据溴桥联配合物 $\left[\mathrm{Cu}_{2} \mathrm{~L}^{3}(\mathrm{Br})\right]^{3+}$ 的晶体结构, $\mathrm{Br}^{-}$包结在配位空腔中并连接两个金属中心，两个金 属离子都是采取变形的三角双锥构型. 卤素离子 与 $\left[\mathrm{Cu}_{2} \mathrm{~L}^{3}\right]^{4+}$ 的键合常数按 $\mathrm{Cl}^{-}>\mathrm{F}^{-}>\mathrm{Br}^{-}>\mathrm{I}^{-}$的顺序递 减, 对 $\mathrm{Cl}^{-}$有一定的选择性(图 9(b)). 穴醚双核铜配合 物 $\left[\mathrm{Cu}_{2} \mathrm{~L}^{3}\right]^{4+}$ 也可以变形为椭球形的配位空腔结合棒 状的三原子阴离子 $\mathrm{N}_{3}{ }^{-}(\log K=4.70(6))$ 和 $\mathrm{NCS}^{-}(\log K=$ $4.28(3))^{[4]}$. 晶体结构分析结果表明 ${ }^{[56]}$, 在 $\mathrm{OH}^{-}$桥联配 合物 $\left[\mathrm{Cu}_{2}(\mathrm{OH}) \mathrm{L}^{3}\right]^{3+}$ 中, $\mathrm{Cu} \cdots \mathrm{Cu}$ 之间的距离是 $3.90 \AA$; 在溴桥联配合物 $\left[\mathrm{Cu}_{2}(\mathrm{Br}) \mathrm{L}^{3}\right]^{3+}$ 中, $\mathrm{Cu} \cdots \mathrm{Cu}$ 之间的距离 是 $4.86 \AA$; 虽然没有得到 $\mathrm{N}_{3}{ }^{-}$和 $\mathrm{NCS}^{-}$桥联配合物的晶 体结构, 但是其 $\mathrm{Cu} \cdots \mathrm{Cu}$ 之间的距离必然会相应增长. 因此, 配合物 $\left[\mathrm{Cu}_{2} \mathrm{~L}^{3}\right]^{4+}$ 具有较强的柔韧性, 可以调整 配位空腔大小包结从 $\mathrm{OH}^{-}$到 $\mathrm{NCS}^{-}$各种不同长度的阴 离子. 这种配位的灵活性和多功能性虽然丰富了阴 离子的配位化学, 但同时也降低了对阴离子的识别 能力.

穴醚配体 $\mathrm{L}^{7}{ }^{[57]}$ 的间隔基团为联苯，其双核铜配 合物 $\left[\mathrm{Cu}_{2}\left(\mathrm{~L}^{7}\right)\left(\mathrm{H}_{2} \mathrm{O}\right)_{2}\right]^{4+}$ (图 10)含有 4 个硝酸根离子, 但 都以抗衡离子的形式存在于配位空腔的外面, 两个 水分子分别与两个金属离子配位形成五配位结构. 双核铜配合物 $\left[\mathrm{Cu}_{2}\left(\mathrm{~L}^{7}\right)\left(\mathrm{H}_{2} \mathrm{O}\right)_{2}\right]^{4+}$ 的配位空腔是一个相 对较长的椭球体，两个水分子氧之间的距离为 $7.36 \AA$, 适于结合较长的阴离子. 例如, 对于一系列位置异构 体如邻、间、对苯二甲酸根阴离子，只有对苯二甲酸 根阴离子与 $\left[\mathrm{Cu}_{2}\left(\mathrm{~L}^{7}\right)\right]^{4+}$ 显示出很强的结合作用. 这是 因为对苯二甲酸根阴离子两个配位的氧原子之间的 距离为 $7.39 \AA$, 很接近 $\left[\mathrm{Cu}_{2}\left(\mathrm{~L}^{7}\right)\left(\mathrm{H}_{2} \mathrm{O}\right)_{2}\right]^{4+}$ 中两个配位 


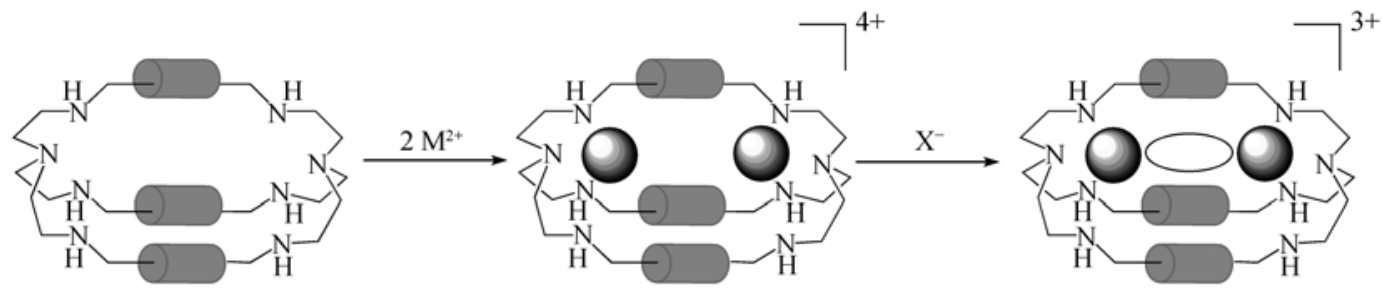

$\bigcirc=\mathrm{M}^{2+} \longrightarrow=\mathrm{x}^{-}$

图 6 氮杂穴醚双核金属配合物与阴离子的结合过程示意图

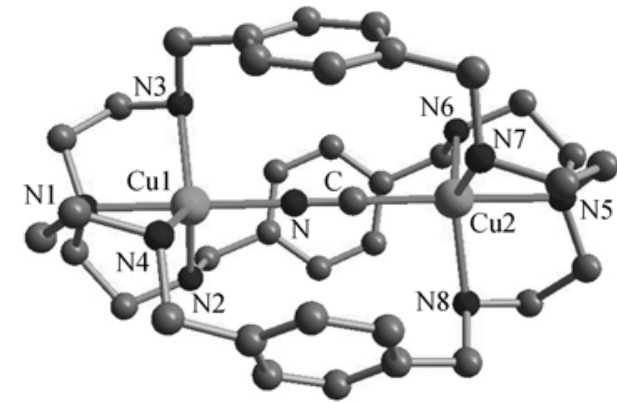

(a)

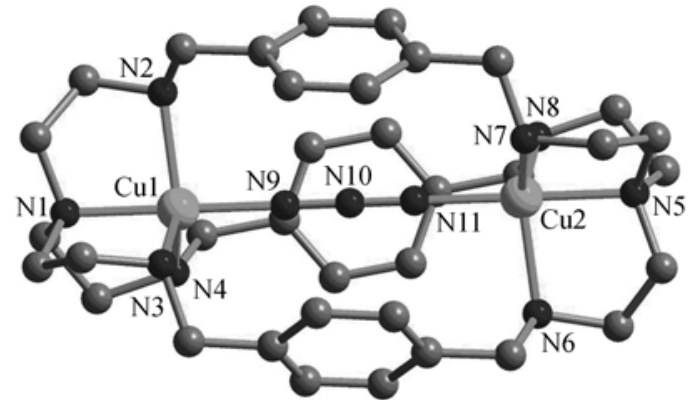

(b)

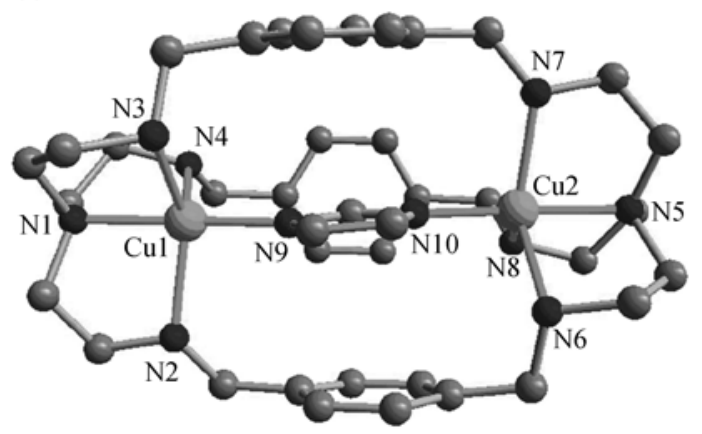

(c)

图 $7\left[\mathrm{Cu}_{2} \mathrm{~L}^{1}\right]^{4+}$ 结合阴离子的晶体结构图

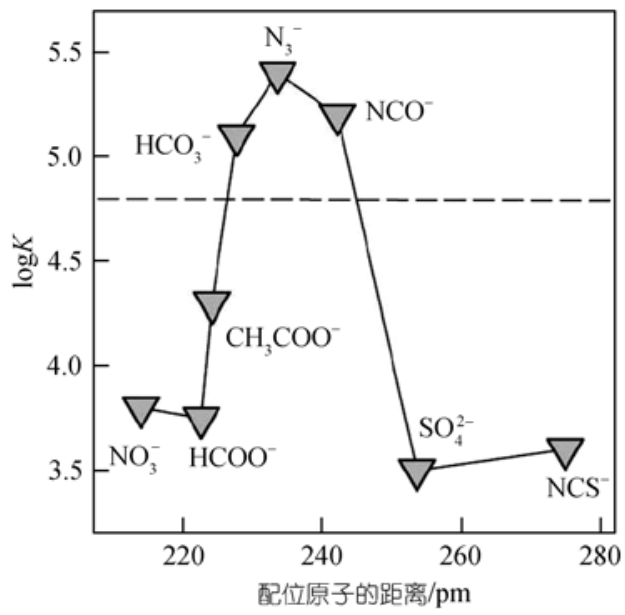<smiles>ON1[14CH2]O[14CH2][14CH2]O1</smiles>

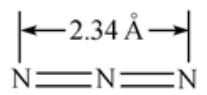

图 $\left.8 \mathrm{Cu}_{2} \mathrm{~L}^{2}\right]^{4}$ 对各种阴离子的识别作用 

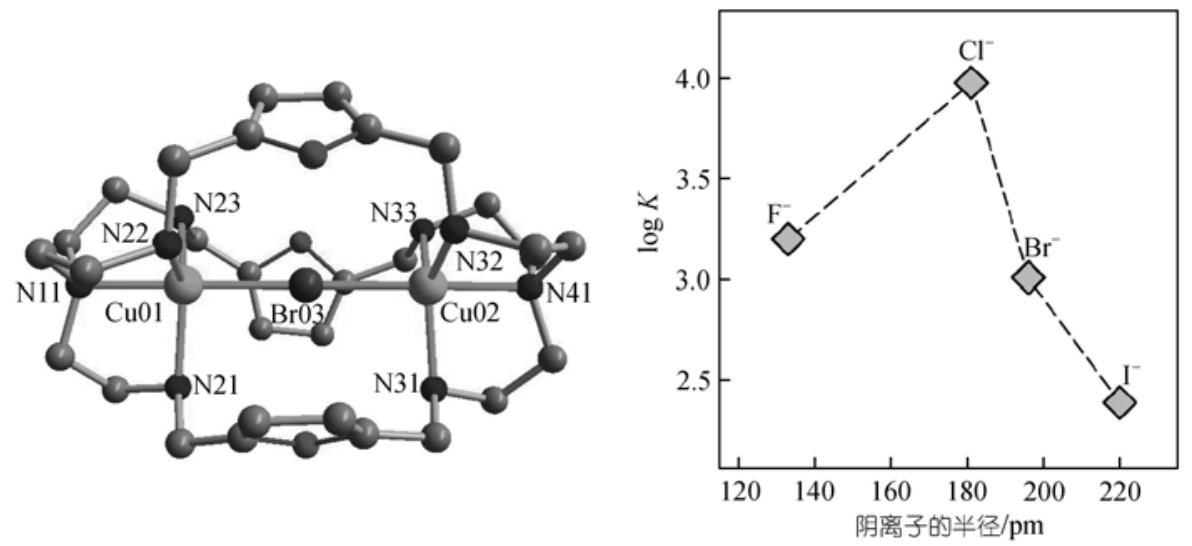

图 $9\left[\mathrm{Cu}_{2} \mathrm{~L}^{3}\right]^{4+}$ 对卤素离子的识别作用

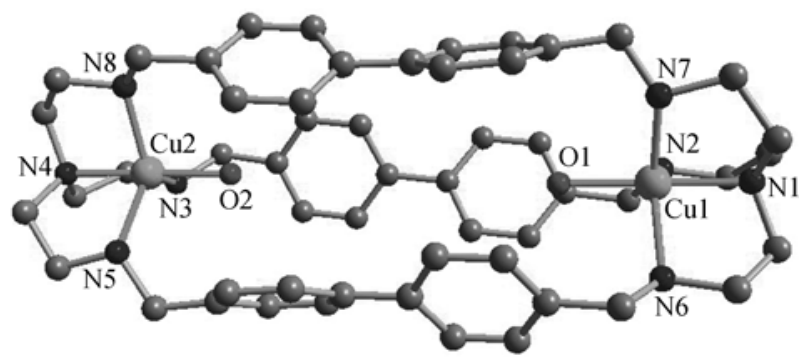

图 $10\left[\mathrm{Cu}_{2}\left(\mathrm{~L}^{7}\right)\left(\mathrm{H}_{2} \mathrm{O}\right)_{2}\right]^{4+}$ 的晶体结构图

氧原子之间的距离 $7.36 \AA$, 因此对苯二甲酸根与两个 金属配位时不会引起配体骨架发生扭曲变形. 相比 之下，邻、间苯二甲酸根阴离子的 $\mathrm{O} \ldots \mathrm{O}$ 距离太短(分 别为 4.98 和 $3.16 \AA$ ), 当其与两个金属中心配位时必 然会导致配体骨架严重扭曲变形，故其结合常数大 大降低. 另外, 当与一系列脂肪族二羧酸根阴离子 $\mathrm{COO}^{-}\left(\mathrm{CH}_{2}\right)_{n} \mathrm{COO}^{-}(n=0 \sim 5)$ 结合时, $\left[\mathrm{Cu}_{2}\left(\mathrm{~L}^{5}\right)\right]^{4+}$ 对戊二 酸根 $(n=3)$ 和己二酸根 $(n=4)$ 表现出很强的选择性. 这同样是由于戊二酸根和己二酸根桥联的双核铜配 合物的 $\mathrm{O} \ldots \mathrm{O}$ 距离最接近 $7.36 \AA$. 而 $\mathrm{O} \ldots \mathrm{O}$ 距离明显
偏离该值的二羧酸根(如丁二酸根, $n=2$; 庚二酸根, $n=5$ )则表现出很弱的配位稳定性. 此外, $\left[\mathrm{Cu}_{2}\left(\mathrm{~L}^{7}\right)\right]^{4+}$ 对一系列氨基酸酯的识别作用也得到了较为系统的 研究 ${ }^{[58]}$. 由于同样的原因 $\left[\mathrm{Cu}_{2}\left(\mathrm{~L}^{7}\right)\right]^{4+}$ 对 $\mathrm{L}$-谷氨酸酯有 很强的识别作用, 其结合常数 $(\log K=6.9(2))$, 明显 高于其他的氨基酸酯.

将苂光基团葸引入到 $\mathrm{L}^{2}$ 的一个间隔基团得到具 有苂光性质的分子识别主体 $\mathrm{L}^{8}{ }^{[59]}$. 由于 $\mathrm{Cu}^{2+}$ 会通过 电荷转移或电子能量转移作用使配体的苂光发生猝 灭，所以不宜采用. 在 $3 \mathrm{~d}$ 过渡金属中，只有 $\mathrm{Zn}^{2+}$ 具有 以下特点: (1) 氧化还原惰性, 不会发生电荷转移作 用; (2) 拥有 $\mathrm{d}^{10}$ 电子构型, 不会产生电子能量转移作 用. 此外, $\mathrm{Zn}^{2+}$ 与 $\mathrm{Cu}^{2+}$ 一样倾向于生成五配位结构, 所以选用 $\mathrm{Zn}^{2+}$ 作为金属中心用于苂光光谱测定法研 究与阴离子的识别作用. 与具有相似结构的双核铜 配合物 $\left[\mathrm{Cu}_{2}\left(\mathrm{~L}^{2}\right)\right]^{4+}$ 类似, $\left[\mathrm{Zn}_{2}\left(\mathrm{~L}^{8}\right)\right]^{4+}$ 对 $\mathrm{N}_{3}{ }^{-}($图 11$)$ 和 $\mathrm{NCO}^{-}$具有较好的识别作用.

氮杂穴醚双核金属配合物是一类新型多功能的 阴离子识别主体，具有不同空间构型的氮杂穴醚配
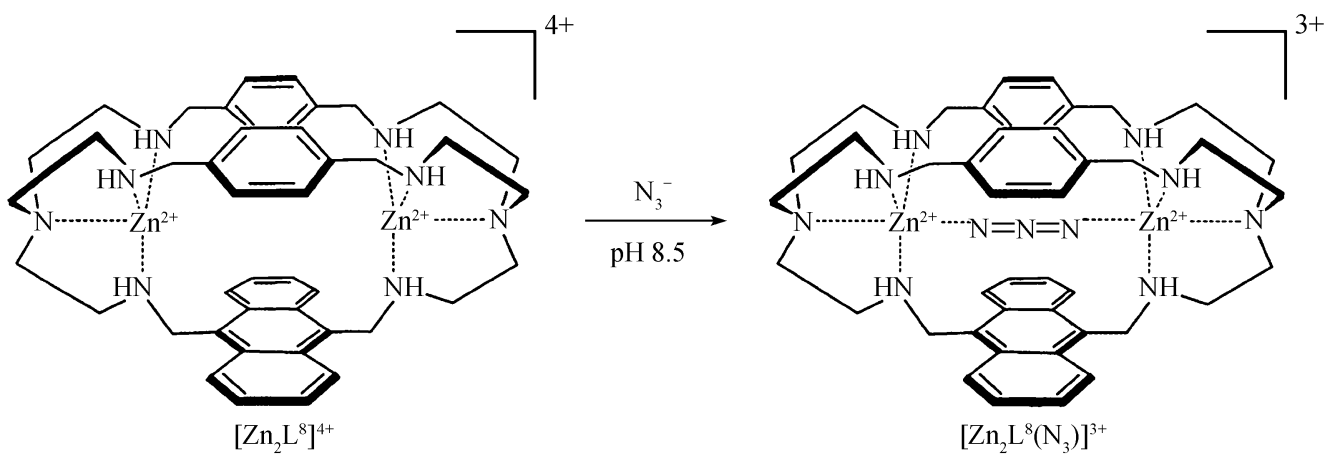

图 $11\left[\mathrm{Zn}_{2} \mathrm{~L}^{8}\right]^{4+}$ 对 $\mathrm{N}_{3}$ 的选择性识别作用 
体与金属离子形成的双核配合物可识别不同的阴离 子, 因此可用来选择性地传输, 回收有用的阴离子或 者去除各种有害的阴离子.

\section{4 氮杂穴醚金属配合物对小分子的活化作用}

氮杂穴醚双核金属配合物对于阴离子的强识别 作用还有可能活化断裂与该阴离子相连的化学键 (图 12). 金属配合物对碳-碳键的活化作用一直是金 属有机化学研究领域中具有挑战性的研究课题 $[60,61]$. 2004 年我们研究发现穴醚双核铜配合物 $\left[\mathrm{Cu}_{2}\left(\mathrm{~L}^{1}\right)\right]^{4+}$ 在 室温下能够高效活化乙腈碳-碳键断裂 ${ }^{[62]}$. 紫外光谱 和质谱数据表明，反应起始物为不含桥联配体的双 核铜配合物 $\left[\mathrm{Cu}_{2}\left(\mathrm{~L}^{1}\right)\right]^{4+}, \mathrm{Cu}(\mathrm{II})$ 离子为变形四面体构 型. 将配合物溶于乙腈后, $\left[\mathrm{Cu}_{2}\left(\mathrm{~L}^{1}\right)\right]^{4+}$ 逐步转化为氧 根桥联的双核配合物 $\left[\mathrm{Cu}_{2}\left(\mathrm{~L}^{1}\right)(\mathrm{CN})\right]^{3+}, \mathrm{Cu}(\mathrm{II})$ 由变形 四面体构型转变为三角双雉构型. 气相色谱检测到 在 $\left[\mathrm{Cu}_{2}\left(\mathrm{~L}^{1}\right)\right]^{4+}$ 与乙腈的反应液中还有甲醇生成. 综合 以上的实验结果, 我们给出乙腈碳-碳键可能的活化 断裂机理(图 13). 根据这一机理, 乙腈的氮原子提供 一对电子与一个 $\mathrm{Cu}(\mathrm{II})$ 离子配位, 同时另一个 $\mathrm{Cu}(\mathrm{II})$ 离子与乙腈 $\mathrm{sp}$ 杂化的碳原子的p轨道作用, 使得电子 从碳转移到 $\mathrm{Cu}(\mathrm{II})$, 从而削弱了与氰根相连的碳-碳 键, 同时使甲基碳的亲电性增强, 受到水分子进攻后, 乙腈碳一碳键发生断裂, 生成甲醇和氰根桥联的双核 铜配合物. 在同样的条件下, $[\mathrm{Cu}($ tren $)]\left(\mathrm{ClO}_{4}\right)_{2}$ 与乙腈 没有类似的反应发生, 这表明穴醚双核铜配合物对 氰根离子的识别作用促使其活化乙腈碳-碳断裂.
进一步研究表明, 双核铜配合物 $\left[\mathrm{Cu}_{2}\left(\mathrm{~L}^{1}\right)\right]^{4+}$ 也能活化 其他脂肪族和芳香族腈化物碳一碳键使之断裂, 其他 双核金属配合物也表现出类似的活化断裂特性. 量 化计算结果表明，在氧根桥联双核金属配合物中，氰 根与 3 个苯环之间有电子云重叠, 表明氧根与苯环 之间存在较强的相互作用. 正是由于 $\left[\mathrm{M}_{2}\left(\mathrm{~L}^{1}\right)\right]^{4+}$ 对氰 根这种强识别作用才导致与之相连的碳-碳键活化并 断裂.

金属配合物对 $\mathrm{CO}_{2}$ 的吸收与转化研究, 不但对于 理解和模拟绿色植物的光合作用 ${ }^{[63]}$ 有指导意义, 而 且对于利用 $\mathrm{CO}_{2}$ 作为原料生产含碳类化工产品有巨 大的应用前景 ${ }^{[64]}$. 同时, $\mathrm{CO}_{2}$ 的吸收与转化对于减少 温室气体对大气环境的影响具有重要意义. 通常情 况下, 金属配合物在中性或碱性溶液中能够吸收空 气中 $\mathrm{CO}_{2}{ }^{[65 ~ 72]}$. 最近, 我们研究发现配合物 $\left[\mathrm{Cu}_{2} \mathrm{~L}^{2}\right]-$ $\left(\mathrm{ClO}_{4}\right)_{4}$ 在室温和弱酸性条件下能够很快吸收空气中 的 $\mathrm{CO}_{2}$, 形成碳酸氢根桥联的双核铜配合物. 碳酸氢 根桥联双核铜配合物能够进一步与一级醇反应生成 碳酸单酯桥联配合物 $\left[\mathrm{Cu}_{2} \mathrm{~L}^{2}\left(\mathrm{CO}_{3} \mathrm{R}\right)\right]\left(\mathrm{ClO}_{4}\right)_{3}(\mathrm{R}=\mathrm{Me}$, $\mathrm{Et}, \mathrm{Pr}, \mathrm{Bu})$. 结合实验和化学理论计算的结果, 我们 提出了 $\left[\mathrm{Cu}_{2} \mathrm{~L}^{2}\right]\left(\mathrm{ClO}_{4}\right)_{4}$ 吸收和转化二氧化碳可能的反 应机理(图 14). 首先在 $\mathrm{pH} \geq 5$ 时, 一个 $\mathrm{OH}^{-}$与双核铜 配合物配位 ${ }^{[22]}$, 接着 $\mathrm{OH}^{-}$进攻二氧化碳的碳原子生 成碳酸氢根桥联的配合物, 碳酸氢根与双核铜配合 物配位后其碳原子的电正性进一步增强, 受到醇的 烷氧基进攻后, 脱去一分子水生成碳酸单酯桥联配 合物. 但是二级和三级醇在相同的条件下基本上不

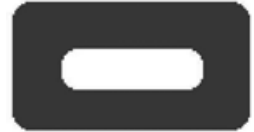

主体

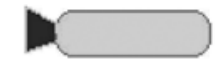

反应物
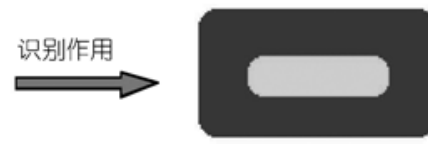

主-客体结合物

\section{反应产物}

图 12 主体对客体分子的识别作用引起与客体分子相连的化学键断裂示意图

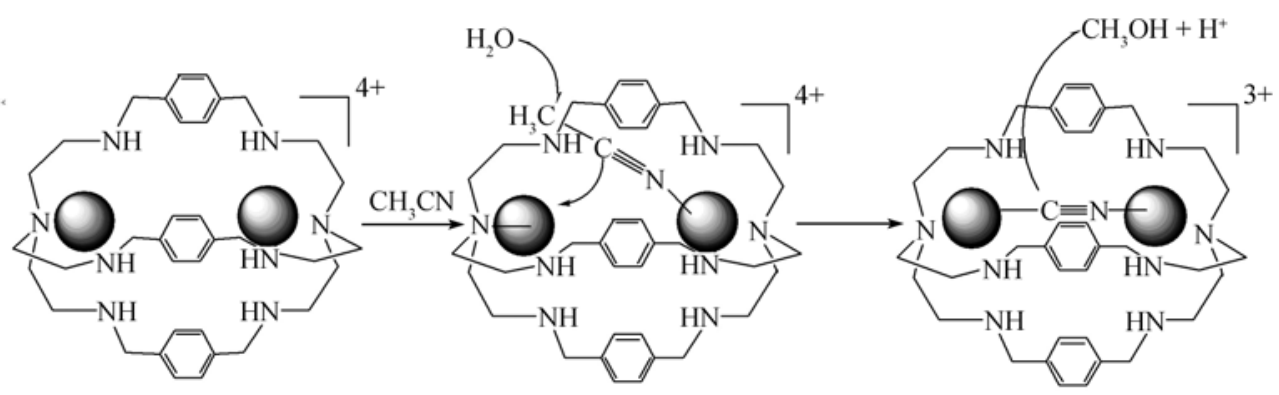

图 $\left.13{ }^{n g}\left[\mathrm{Cu}_{2} \mathrm{~L}^{\mathrm{a}}\right)\right]^{4+}$ 活化乙腈碳-碳键断裂机理示意图 

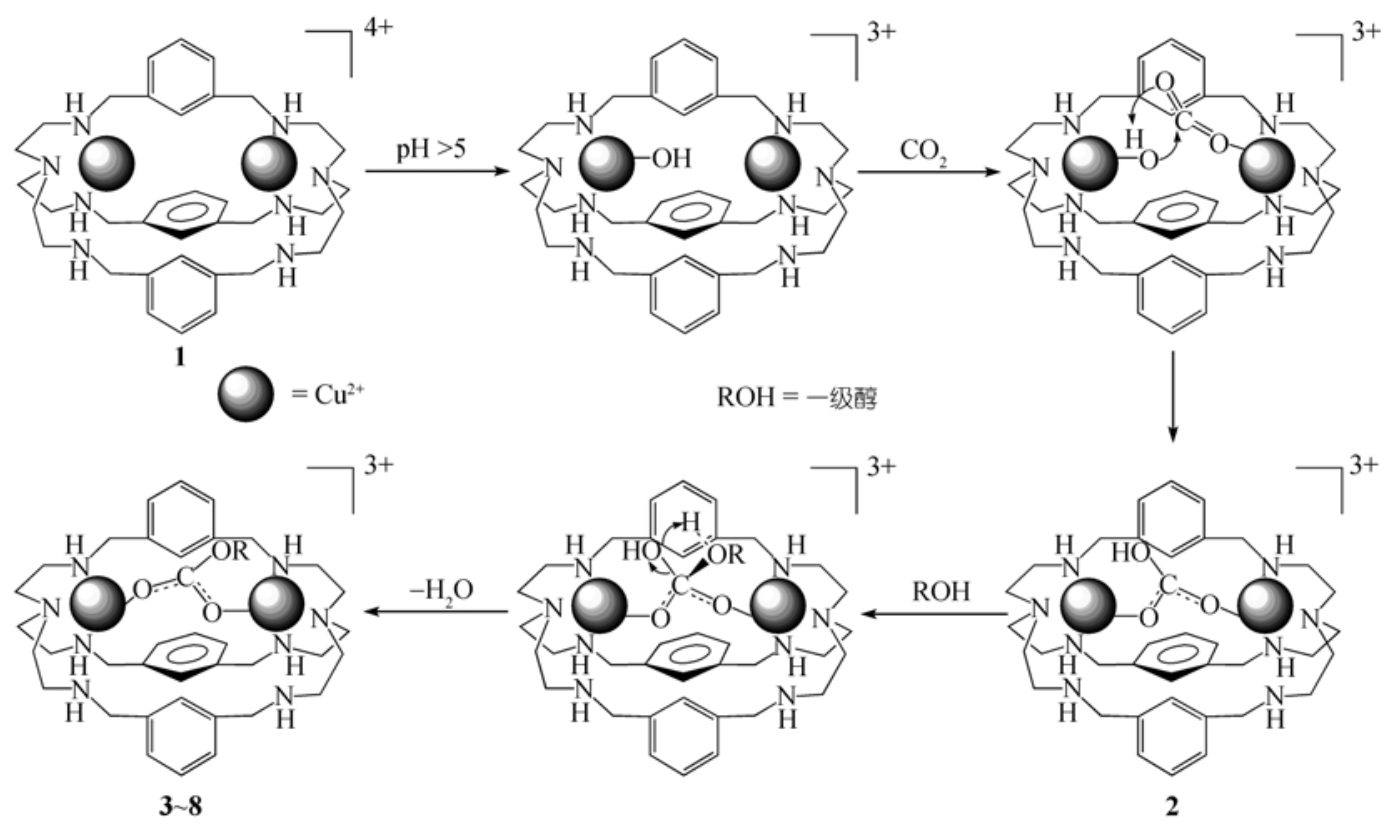

图 $14\left[\mathrm{Cu}_{2}\left(\mathrm{~L}^{2}\right)\right]^{4+}$ 吸收与活化 $\mathrm{CO}_{2}$ 催化机理的示意图

发生反应. 穴醚配体 $\mathrm{L}^{2}$ 的间苯基团的空间位阻效应 可能是造成上述反应活性差异的主要原因.

虽然穴醚 $\mathrm{L}^{1}$ 与 $\mathrm{L}^{2}$ 的结构相似, 但其双核铜配合 物 $\left[\mathrm{Cu}_{2}\left(\mathrm{~L}^{1}\right)\right]^{4+}$ 和 $\left[\mathrm{Cu}_{2}\left(\mathrm{~L}^{2}\right)\right]^{4+}$ 的反应性能有很大差别. 这主要是由于 $\mathrm{L}^{1}$ 的刚性结构使 3 个苯环相互靠近, 与外界交换的 窗口”较小, 只能让线形分子进入, 故 $\left[\mathrm{Cu}_{2}\left(\mathrm{~L}^{1}\right)\right]^{4+}$ 不吸收空气中 $\mathrm{CO}_{2}$, 轴向位置空缺的金属 离子可以与腈化物作用并使其碳-碳键活化断裂. 在 $\mathrm{L}^{2}$ 中, 3 个苯环可以旋转一定角度使其“窗口”变大, 因 此能够识别 $\mathrm{HCO}_{3}{ }^{-}$这种较大的阴离子, 导致 $\left[\mathrm{Cu}_{2}\left(\mathrm{~L}^{2}\right)\right]^{4+}$ 非常容易吸收空气中的 $\mathrm{CO}_{2}$ 并转化为碳酸酯. 这充 分体现了氮杂穴醚大环金属配合物结构的微小差异 即可导致其对小分子的识别作用与活化作用的巨大 差别.

\section{5 展望}

氮杂穴醚配体及其双核金属配合物在小分子识 别方面具有特殊的功能, 是主客体化学的重要研究 内容. 如前所述, 氮杂穴醚配体的仲胺原子容易质子 化，质子化后的配体与其金属配合物都具有一个正 电性的空腔, 在溶液中非常容易选择性键合有害的 阴离子, 并能快速固定空气中的二氧化碳, 这将有助 于人们监控和去除环境中的有害阴离子, 如 $\mathrm{NO}_{3}^{-}$, $\mathrm{PO}_{4}{ }^{3-}, \mathrm{AlO}_{3}{ }^{3-}$ 等, 及燃料燃烧放出的大量二氧化碳气 体，从而减小“温室效应”所带来的严重后果. 氮杂
穴醚双核金属配合物在基于分子识别的基础上对不 同小分子具有活化作用，这方面工作的深入开展在 理论研究和实际应用中都具有较好的前景. 另外, 氮 杂穴醚双核金属配合物具有与生物体内许多金属酶 类似的双金属中心结构, 为我们提供了大量人工模 拟天然酶活性中心的模型，对其结构与功能的研究, 将有助于我们更深地了解金属酶的催化机理.

\section{参考文献}

1 Lehn J M. Cryptates: the chemistry of macropolycyclic inclusion complexes. Acc Chem Res, 1978, 11(2): 49-57

2 Llinares M J, Powell D, Bowman-James K. Ammonium based anion receptors. Coord Chem Rev, 2003, 240(1): 57-75

3 McKee V, Nelson J, Town R M. Caged oxoanions. Chem Soc Rev, 2003, 32(5): 309-325[DOI]

4 Kang S O, Begum R A, Bowman-James K. Amide-based ligands for Anion Coordination. Angew Chem Int Ed, 2006, 45: 7882-7894[DOI]

5 Amendola V, Bonizzoni M, Fabbrizzi L, et al. Some guidelines for the design of anion receptors. Coord Chem Rev, 2006, 250: 14511470 [DOI]

6 O'Neil E J, Smith B D. Anion recognition using dimetallic coordination complexes. Coord Chem Rev, 2006, 250: 3068-3080[DOI]

7 Lehn J M. Supramolecular chemistry-molecules, supermolecules, and molecular functional units. Angew Chem, 1988, 100: 91-116

8 Lehn J M. Perspectives in supramolecular chemistry: from molecular recognition to molecular information processing and self organization. Angew Chem, 1990, 102: 1347-1362

9 Katayev E A, Ustynyuk Y A, Sessler J L. Receptors for tetrahedral oxyanions. Coord Chem Rev, 2006, 250: 3004-3007[DOI] 
10 Kang S O, Hossain M A, Bowman-James K. Influence of dimensionality and charge on anion binding in amide-based macrocyclic receptors. Coord Chem Rev, 2006, 250: 3038-3052[DOI]

11 Wichmann K, Antonioli B, Gloe K, et al. Polyamine-based anion receptors: Extraction and structural studies. Coord Chem Rev, 2006, 250: 2987-3003[DOI]

12 Wilcox D E. Binuclear metallohydrolases. Chem Rev, 1996, 96(7): $2435-2458[\mathrm{DOI}]$

13 Pierre J L, Chautemps P, Refaif S, et al. Imidazolate-bridged dicopper( II) and copper-zinc complexes of a macrobicyclic lignad (cryptand). A possible model for the chemistry of $\mathrm{Cu}-\mathrm{Zn}$ superoxide dismutase. J Am Chem Soc, 1995, 117(7): 1965-1973[DOI]

14 Chen D, Martell A E. The synthesis of new binucleating polyaza macrocyclic and macrobicyclic ligands: dioxygen affinities of the cobalt complexes. Tetrahedron, 1991, 47: 6895-6902[DOI]

15 Motekaitis R J, Martell A E, Lehn J M, et al. Anion binding in macrobicyclic metal cryptate complexes: copper( II )-bistren. Inorg Chem, 1984, 23(11): 1588-1591[DOI]

16 Motekaitis R J, Martell A E, Lehn J M, et al. Comparative study of the copper( II ) cryptates of C-BISTREN and O-BISTREN. Protonation constants, formation constants, and secondary anion bridging by fluoride and hydroxide. Inorg Chem, 1988, 27(20): 3630 - 3636[DOI]

17 Hossain M A, Llinares J M, Bowman-James K, et al. Further insight to selectivity issues in halide binding in a tiny octaazacryptand. Chem Commun, 2000, 2269-2270

18 Coyle J, Drew M G B, Nelson J, et al. Expanded -cap sepulchrates: a new series of mononuclear transition-metal ion cryptates. Dalton Trans, 1997, 1123-1125

19 Farrar J A, Grinter R, Thomson A J, et al. The electronic structure of the mixed-valence copper dimer $\left[\mathrm{Cu}_{2}\left\{\mathrm{~N}\left(\mathrm{CH}_{2} \mathrm{CH}_{2} \mathrm{~N}=\mathrm{CHCH}=\right.\right.\right.$ $\left.\left.\left.\mathrm{NCH}_{2} \mathrm{CH}_{2}\right)_{3} \mathrm{~N}\right\}\right]^{3+}$. Dalton Trans, 1997, 4083-4087

20 Coyle J L, McKee V, Nelson J. Response to steric constraint by $\mathrm{d}^{10}$ cations: an 'A-frane' disilver cryptate. Chem Commun, 1998, 709-710

21 Hirschheydt V T, Wolfart V, Gleiter R, et al. A short survey of bicyclic diamines-syntheses and properties of N,N'-bridged-1,10- diazabicyclooctadeca-5,14-diynes. Perkin Trans, 2000, 2, 175-183[DOI]

22 Menif R, Reibenspies J, Martell A E. Synthesis, protonation constants, and copper( II) and cobalt( II ) binding constants of a new octaaza macrobicyclic cryptand: $(\mathrm{MX})_{3}(\mathrm{TREN})_{2}$. Hydroxide and carbonate binding of the dicopper( II) cryptate and crystal structures of the cryptand and of the carbonato-bridged dinuclear copper( II ) cryptate. Inorg Chem, 1991, 30(18): 3446-3454[DOI]

23 Deeney F A, Harding C J, Morgan G G, et al. Response to steric constraint in azacryptate and related complexes of iron-(II) and -(III). Dalton Trans, 1998, 1837-1843

24 Escuer A, Harding C J, Dussart Y, et al. Constrained ferromagnetic coupling in dinuclear $\mu_{1,3}$-azido nickel(II) cryptate compounds. Crystal structure and magnetic behaviour of $\left[\mathrm{Ni}_{2}(\mathrm{~L} 1)\left(\mathrm{N}_{3}\right)\left(\mathrm{H}_{2} \mathrm{O}\right)\right]$ $\left[\mathrm{CF}_{3} \mathrm{SO}_{3}\right]_{3} \cdot 2 \mathrm{H}_{2} \mathrm{O} \cdot \mathrm{EtOH}\left\{\mathrm{Ll}=\mathrm{N}\left[\left(\mathrm{CH}_{2}\right)_{2} \mathrm{NHCH}_{2}\left(\mathrm{C}_{6} \mathrm{H}_{4}-m\right) \mathrm{CH}_{2} \mathrm{NH}\left(\mathrm{CH}_{2}\right)_{2}\right]_{3} \mathrm{~N}\right\}$. Dalton Trans, 1999, 223-227

25 Adams H, Elsegood M R J, Fenton D E, et al. Dinuclear silver( I ) complexes of bibracchial tetraimine Schiff base macrocycles derived from pyrrole-2,5-dicarbaldehyde. Dalton Trans, 1999, $2031-$
2037

26 Platas C, Avecilla F, Rodríguez-Blas T, et al. Mono- and bimetallic lanthanide( III) phenolic cryptates obtained by template reaction: solid state structure, photophysical properties and relaxivity. Dalton Trans, 2000, 611-618

27 Xiong R G, Zhang J, You X Z, et al. In situ ligand synthesis and the first crystallographically characterized lanthanide 3-D pillared networks containing benzene-1,4-disulfonate as a building block. Dalton Trans, 2001, 780-782

28 Chen Q Y, Luo Q H, Fu D G, et al. A study on the heterodinuclear cryptates $[\mathrm{LnCuL}(\mathrm{DMF})]\left(\mathrm{ClO}_{4}\right)_{2} \cdot \mathrm{MeCN}(\mathrm{Ln}=\mathrm{Gd}, \mathrm{Eu}, \mathrm{Tb}, \mathrm{Dy}, \mathrm{Y})$ synthesis, characterization, magnetic and electrochemical properties. Dalton Trans, 2002, 2873-2878

29 Farrell D, Gloe K, Goretzke G, et al. Towards promising oxoanion extractants: azacages and open-chain counterparts. Dalton Trans, 2003, $1961-1968$

30 Bazzicalupi $\mathrm{C}$, Bencini A, Bianchi A, et al. $\mathrm{pH}$ modulation of the luminescence emission of a new europium cryptate complex. Chem Commun, 2000, 561-562

31 Lamarque L, Miranda C, Navarro P, et al. Dopamine interaction with a polyamine cryptand of $1 \mathrm{H}$-pyrazole in the absence and in the presence of $\mathrm{Cu}(\mathrm{II})$ ions. Crystal structure of $\left[\mathrm{Cu}_{2}\left(\mathrm{H}_{-1} \mathrm{~L}\right)\right]$ $\left(\mathrm{ClO}_{4}\right)_{3} \cdot 2 \mathrm{H}_{2} \mathrm{O}$. Chem Commun, 2000, 1337-1338

32 Mukhopadhyay P, Bharadwaj P K, Savitha G, et al. The first D- $\pi$-A octupolar cryptand molecule to exhibit bulk non-linearity. Chem Commun, 2000, 1815-1816

33 Chen Q Y, Feng C J, Luo Q H, et al. The syntheses and luminescent properties of $\mathrm{Eu}(\mathrm{III})$ and $\mathrm{Tb}(\mathrm{III})$ cryptates in solution. Eur J Inorg Chem, 2001, 1063-1069

34 Chen Q Y, Luo Q H, Hu X L, et al. Heterodinuclear cryptates $[\operatorname{EuML}(\mathrm{dmf})]\left(\mathrm{ClO}_{4}\right)_{2}(\mathrm{M}=\mathrm{Ca}, \mathrm{Cd}, \mathrm{Ni}, \mathrm{Zn})$ : tuning the luminescence of europium( III) through the selection of the second metal ion. Chem Eur J, 2002, 8: 3984-3990[DOI]

35 Ma Z, Yang R D, Yan L. New cryptands synthesized by $[2+3]$ condensation and reduction with or without templates. J Chem Res-S, 1999, 712-713

36 Das G, Tripathi P, Bharadwaj K P, et al. Facile one-pot synthesis of macrobicyclic/macrotricyclic cryptands: effect of reactant concentrations. Tetrahedron, 2000, 56(11): 1501 - 1504[DOI]

37 Hossain A M, Morehouse P, Bowman-James K, et al. Tritopic (cascade) and ditopic complexes of halides with an azacryptand. Inorg Chem, 2005, 44(7): 2143-2149[DOI]

38 Lakshminarayanan P S, Kumar K D, Ghosh P. Counteranion- controlled water cluster recongnition in a protonated octaamino cryptand. Inorg Chem, 2005, 44(21): 7540-7546[DOI]

39 Ravikumar I, Lakshminarayanan P S, Ghosh P, et al. Recognition of water-acetonitrile-water cluster in a tetraprotonated picrate salt of octaaminocryptand. Crystal Growth \& Design, 2006, 6(12): $2630-2633$ [DOI]

40 Mason S, Llinares M J, Bowman-James K, et al. Snapshots of fluoride binding in an aza cryptand. J Am Soc Chem, 2000, 122(8): $1814 \sim 1815[\mathrm{DOI}$

41 Mason S, Clifford T, Bowman-James K, et al. Unusual encapsula- 
tion of two nitrates in a single bicyclic cage. J Am Soc Chem, 1998, 120(34): 8899-8900[DOI]

42 Hynes J M, Maubert B, McKee V, et al. Protonated azacryptate hosts for nitrate and perchlorate. Dalton Trans, 2000, 2853-2859

43 Kang O S, Hossain A M, Bowman-James K, et al. Encapsulated sulfates: insight to binding propensities. Chem Commun, 2005, $328-330$

44 Maubert M B, Nelson J, McKee V, et al. Selectivity for dinegative versus mononegative oxoanionic guests within a cryptand host. Dalton Trans, 2001, 1395-1397

45 Nelson J, Nieuwenhuyzen M, Pál I, et al. Dual-mode recognition of oxalate by protonated azacryptate hosts; conformational response of the guest maximizes $\pi$-stacking interactions. Chem Commun, 2002, 2266-2267

46 Morgan G, McKee V, Nelson J. Caged anions: perchlorate and perfluoroanion cryptates. Chem Commun, 1995, 1649-1652

47 McKee V, Morgan G G. A perchorate salt of an octaprotonated cryptand. Acta Cryst, 2003, C59(3): 150-152

48 Lehn J M, Meric R, Vigneron J P, et al. Molecular recognition of anionic substrates. Binding of carboxylates by a macrobicyclic coreceptor and crystal structure of its supramolecular cryptate with the terephthalate dianion. Chem Commun, 1991, 62-64

49 Teulade-Fichou M P, Vigneron J P, Lehn J M. Detection of organic anions in water through complexation enhanced fluorescence of a macrobicyclic tris-acridine cryptand. J Chem Soc Perkin Trans, 1996, 2(10): 2169-2175

50 Lehn J M. Supramolecular chemistry: receptors, catalysts, and carriers. Science, 1985, 227: 849-856[DOI]

51 Bond D A, Derossi S, Nelson J, et al. Cascade complexation: a single cyano bridge links a pair of $\mathrm{Cu}(\mathrm{II})$ cations. Dalton Trans, 2005, 2403-2409

52 Ravikumar I, Suresh E, Ghosh P. A perfect linear Cu-NNN-Cu unit inside the cryptand cavity and perchlorate entrapment within the channel formed by the cascade complex. Inorg Chem, 2006, 45(25): 10046-10048[DOI]

53 Zhuang X M, Lu T B, Chen S. Cyanide and imidazolate bridged macrocyclic dinuclear $\mathrm{Cu}(\mathrm{II})$ complexes: Synthesis, structure and magnetic properties. Inorg Chim Acta, 2005, 358: 2129-2134[DOI]

54 Fabbrizzi L, Leone A, Taglietti A. A chemosensing ensemble for selective carbonate detection in water based on metal- ligand interations. Angew Chem Int Ed, 2001, 40: 3066-3069[DOI]

55 Amendola V, Bastianello E, Fabbrizzi L, et al. Halide-ion encapsulation by a flexible dicopper( II ) bis-tren cryptate. Angew Chem Int Ed, 2000, 39(16): 2917-2920[DOI]

56 Harding C J, McKee V, Nelson J, et al. Linear Cu-OH-Cu: magnetic silence for dicopper( II) in trigonal bipyramidal coordination geometry. Chem Commun, 1993, 1768-1770

57 Boiocchi M, Bonizzoni M, Fabbrizzi L, et al. A dimetallic cage with a long ellipsoidal cavity for the fluorescent detection of dicarboxylate anions in water. Angew Chem Int Ed, 2004, 43: $3847-$ 3852[DOI]

58 Bonizzoni M, Fabbrizzi L, Piovani G, et al. Fluorescent detection of glutamate with a dicopper( II) polyamine cage. Tetrahedron, 2004, 60: $11159-11162$
59 Fabbrizzi L, Faravelli I, Francese G, et al. A fluorescent cage for anion sensing in aqueous solution. Chem Commun, 1998, 971-972

60 Taw F L, White P S, Bergman R G, et al. Carbon-carbon bond activation of $\mathrm{R}-\mathrm{CN}\left(\mathrm{R}=\mathrm{Me}, \mathrm{Ar},{ }^{\mathrm{i}} \mathrm{Pr},{ }^{\mathrm{t}} \mathrm{Bu}\right)$ using a cationic $\mathrm{Rh}(\mathrm{III})$ complex. J Am Chem Soc, 2002, 124(16): 4192-4193[DOI]

61 Taw F L, Mueller A H, Bergman R G, et al. A mechanistic investigation of the carbon-carbon bond cleavage of aryl and alkyl cyanides using a cationic Rh(III) silyl complex. J Am Chem Soc, 2003, 125(32): 9808-9813[DOI]

$62 \mathrm{Lu} \mathrm{T} \mathrm{B,} \mathrm{Zhuang} \mathrm{X} \mathrm{M,} \mathrm{Li} \mathrm{Y} \mathrm{W,} \mathrm{et} \mathrm{al.} \mathrm{C-C} \mathrm{bond} \mathrm{cleavage} \mathrm{of} \mathrm{acetoni-}$ trile by a dinuclear copper( II ) cryptate. J Am Chem Soc, 2004, 126(15): 4760-4761[DOI]

63 Cleland W W, Andrews T J, Lorimer G H, et al. Mechanism of Rubisco: The carbamate as general base. Chem Rev, 1998, 98(2): $549-561[\mathrm{DOI}]$

64 Inoue S, Yamazaki N, eds. Organic and Bioorganic Chemistry of Carbon Dioxide. Tokyo: Kodansha, 1981

65 Kong L Y, Zhu H F, Sun W Y, et al. Copper(II) and zinc(II) complexes can fix atmospheric carbon dioxide. Angew Chem Int Ed, 2005, 44: 4352-4355[DOI]

66 Kong L Y, Zhu H F, Sun W Y, et al. Cadmium( II ) and copper( II ) complexes with imidazole-containing tripodal polyamine ligands: $\mathrm{pH}$ and anion effects on carbon dioxide fixation and assembling. Inorg Chem, 2006, 45(20): 8098-8107[DOI]

67 Verdejo B, Aguilar J, Espana G, et al. $\mathrm{CO}_{2}$ fixation by $\mathrm{Cu}^{2+}$ and $\mathrm{Zn}^{2+}$ complexes of a terpyridinophane aza receptor. Crystal structures of $\mathrm{Cu}^{2+}$ complexes, pH-metric, spectroscopic, and electrochemical studies. Inorg Chem, 2006, 45(9): 3803-3815 [DOI]

68 Bazzicalupi C, Bencini A, Bencini A, et al. $\mathrm{CO}_{2}$ fixation by novel copper( II) and zinc(II) macrocyclic complexes. A solution and solid state study. Inorg Chem, 1996, 35(19): 5540-5548 [DOI]

69 Escuer A, Mautner F A, Vicente R, et al. Crystal structure and magnetic behavior of two new dinuclear carbonato -bridged copper (II) compounds. Superexchange pathway for the different coordination modes of the carbonato bridge in polynuclear copper( II) compounds. Inorg Chem, 1998, 37(17): 4190-4196 [DOI]

70 Kitajima N, Hikichi S, Tanaka M, et al. Fixation of atmospheric carbon dioxide by a series of hydroxo complexes of divalent metal ions and the implication for the catalytic role of metal ion in carbonic anhydrase. Synthesis, characterization, and molecular structure of $[\mathrm{LM}(\mathrm{OH})]_{\mathrm{n}}(n=1$ or 2$)$ and $\mathrm{LM}\left(-\mathrm{CO}_{3}\right) \mathrm{ML}(\mathrm{M}(\mathrm{II})=\mathrm{Mn}, \mathrm{Fe}$, Co, Ni, Cu, Zn; L = HB(3,5-iso-Pr2pz) $)$. J Am Chem Soc, 1993, 115(13): 5496-5508[DOI]

71 Kato M, Ito T. Facile carbon dioxide uptake by zinc( II ) -tetraazacycloalkane complexes. 1. Syntheses, characterizations, and chemical properties of (monoalkyl carbonato) (tetraazacycloalkane) zinc( II ) complexes. Inorg Chem, 1985, 24(4): 504-508[DOI]

72 Kato M, Ito T. Facile carbon dioxide uptake by zinc( II )- tetraazacycloalkane complexes. 2. X-ray structural studies of (-monomethyl carbonato)(1,4,8,11-tetraazacyclotetradecane) zinc( II ) perchlorate, bis(-monomethyl carbonato)tris $[(1,4,8,12$-tetraazacyclopentadecane) zinc( II )] perchlorate, and (monomethyl carbonato) (1,4, 8,11-tetramethyl-1,4,8,11-tetraazacyclotetradecane) zinc( II ) perchlorate. Inorg Chem, 1985, 24(4): 509-514[DOI] 